\title{
Normal eating is counter-cultural: embodied experiences of eating disorder recovery
}

Andrea LaMarre

University of Guelph

Carla Rice

University of Guelph

This is the peer reviewed version of the following article: LaMarre, A., \& Rice, C. (2015). Normal eating is counter-cultural: Embodied experiences of eating disorder recovery. Journal of Community \& Applied Social Psychology, 26(2): 136-149, which has been published in final form at https://doi.org/10.1002/casp.2240. This article may be used for non-commercial purposes in accordance with Wiley Terms and Conditions for Use of SelfArchived Versions. 


\section{Normal Eating Is Counter-Cultural: Embodied Experiences of Eating Disorder Recovery}

Andrea LaMarre and Carla Rice

\section{ABSTRACT}

Standards for eating disorder recovery, although they originate in lifesaving methods, may be unattainable for certain individuals. We used narrative thematic analysis to explore the stories of 10 young women in eating disorder recovery. Participants' narratives highlight the complexity and counter-cultural nature of navigating prescriptions for recovery in a sociocultural context that privileges some bodies and food-related behaviours over others. Our main themes were materiality of eating disorders recovery, beyond biometrics, embodying counter-culture, embodying recovery and authenticity/invisibility. Our analysis reveals a biopedagogy of eating disorder recovery, which offers individuals in recovery sets of instructions for how to be healthy that run in opposition to dominant prescriptions for health. These findings help us better understand the lived experience of eating disorder recovery, highlighting difficulties of recovering in a culture that moralizes food and bodies. Further investigating how standards for recovery intersect with dominant discourses for healthy living could help to support individuals in recovery.

\section{Keywords}

eating disorders; recovery narratives; feminism; qualitative; biopedagogy 
Eating disorder recovery remains an elusive construct despite decades of research. Studies attempting to quantitatively define recovery have provided insights into the physical (e.g. weight restoration) and psychological (e.g. cognitive flexibility) components of recovery (BardoneCone et al., 2010). Qualitative studies have added to our understanding of eating disorder recovery; for example, unearthing how individuals in recovery and clinicians may hold different perspectives on recovery (Noordenbos \& Seubring, 2006), the various stages of eating disorder recovery from the individual's perspective (Dawson, Rhodes, \& Touyz, 2014) and how people may have difficulty imagining recovery (Malson et al., 2011). However, the normalizing and often moralizing prescriptions for healthy living that underpin the term 'recovery' itself have not featured strongly in attempts to establish a definition of recovery upon which clinicians and people in recovery can agree. In this paper, we ask the following: Can assembling objective criteria for and articulating a privileged version of eating disorder recovery alienate and/or overwhelm some people in recovery? To unpack this question, we draw on recent developments in critical health scholarship on 'biopedagogies' to theorize a biopedagogy of eating disorder recovery.

Biopedagogies are loose collections of information, instructions and directives about how to live, how to be embodied, what 'health' is and what to do in order to be healthy and happy (Harwood, 2010, p. 16); they also contain instructions about how to achieve recovery and wellness (LaMarre \& Rice, in press) and how to avoid 'risk' (Fullagar, 2009, p. 124). Transmitted in formal clinical and educational contexts, in the media and in everyday interactions, many biopedagogical instructions are specifically 
designed to address problems related to eating and weight (Wright \& Harwood, 2009). Teachings around 'how to be healthy' and 'how to be recovered' are grounded in technologies of cure designed to be life saving. However, when recovery prescriptions are mapped onto broader pedagogical instructions for health for the general population (specifically around obesity prevention), the limits, difficulties and paradoxes of eating disorder recovery biopedagogies become clear. Building on the qualitative literature around eating disorder recovery, we situate the embodied experience of eating disorder recovery within a sociocultural context rife with moralizing imperatives around food, health and bodies. Our study adds to this literature, exploring the multi-directional interaction of discourses on health and bodies that individuals confront in recovery.

\section{THE ILLUSIVE CONSTRUCT OF RECOVERY}

Biomedical discourses inform approaches to eating disorder treatment and recovery; most treatment programmes have nutrition and/or weight restoration as a primary goal (Rance, Clarke, \& Moller, 2014). Recovery discourses often employ biomedical parameters, including weight restoration and 'normal eating' (Bardone-Cone et al., 2010; Gremillion, 2003). Long-term outcome studies do not tend to problematize the construct of recovery and may imply that those who no longer fit the Diagnostic and Statistical Manual criteria are recovered regardless of their subjective perspectives.

Oriented to generating in-depth analyses, the qualitative literature offers greater insight into people's experiences of recovery. Exploring the extent to which individuals living with eating disorders imagine recovery, for 
example, Malson et al. (2011), found that people often felt as though those around them subsumed their identities into their eating dis- orders, limiting their ability to imagine what recovery might be like. Darcy et al. (2010) also suggest that even after they have left treatment, people may feel ambivalent about recovery or see it as unachievable. People with eating disorders who feel that they have reached a point of recovery often credit positive interpersonal experiences, such as strong relational ties, with helping them turn the corner to wellness (Dawson et al., 2014; Hay \& Cho, 2013). Recovery is sometimes regarded to those experiencing it as 'becoming whole again' (Jenkins \& Ogden, 2012) or coming to a place of reflection about past experiences (Dawson et al., 2014). These perspectives give us insight into what the recovery experi- ence may be like for some individuals with eating disorders; however, not all people in recovery agree with the idea that recovery entails an overcoming and divorcing of self from eating disorder. Instead, some may think about their subjectivity as 'poly-vocal', that is, as multi-faceted, with various aspects of the self coming to be more or less important at different times (Saukko, 2008).

In addition to offering new perspectives on the recovery experience, these studies reveal how social locations, including ethnicity, socioeconomic status and gender, may play a role in shaping conceptualizations of and desires to achieve recovery (Ison \& Kent, 2010). Building on these studies, our research attends to how sociocontextually located embodied experiences impact people's ability to attain recovery. This facet of recovery may be especially important in a sociohistorical moment marked by extreme scrutiny on bodies and health. Following from 
a 'war on obesity' over the past two decades, fatness is stigmatized, equated with laziness, ill health, ugliness and a lack of restraint and will power (Rice, 2007). We suggest that adding body size to the recovery equation highlights difficulties with following prescriptions for recovery in a society that positions weight gain as wholly negative. Further, the struggle with recovery prescriptions may be compounded for those whose disordered eating is not marked by dramatic weight loss.

\section{DISCOURSES ON THE BODY AND BIOPEDAGOGIES}

Situating eating disorders, disordered eating and recovery within sociocultural and historical context helps us to unpack the instructional messages we receive about bodies, health and possibilities for recovery. Foucault (1979) theorized the operations of power in mod- ern societies, suggesting that citizens' bodies are subject to a diffuse kind of governmental power, 'biopower'. Instead of exerting direct force, biopower operates more subtly; people themselves internalize this kind of power in forming their sense of self and engage in self-monitoring to conform to what is considered normal, such as by adopting normative behaviours around food, weight and health (Foucault, 1979).

Wright and Harwood (2009) take up Foucault's notion of biopower, theorizing a set of biopedagogies that operate around 'obesed' bodies [bodies defined as obese by measures such as body mass index (BMI) and treated as deficient in social contexts]. Transmitted and internalized through formal and informal educational sites, biopedagogies inform people what they must do in order to be healthy and happy (Halse, 2009). These moralizing instructions about bodies and bodily management circulate in 
schools (Evans \& Rich, 2011), doctors' offices (Rail, 2012), public health campaigns (Rice, 2014) and beyond. Biopedagogical messages are tenacious: In a society enamoured of obesity prevention efforts and bent on promoting productive bodies, they give signals for how people might achieve maximum mental and physical fitness to ensure a competitive body politic. Importantly, biopower can have detrimental effects on those who are unable to replicate the expected results of bodywork (i.e. a fit, thin, healthy body).

Although some biopedagogical scholarship alludes to how messages about fit bodies might be implicated in producing disordered eating, to our knowledge, there has yet to be significant theorizing around the idea of a biopedagogy specifically related to eating disorder recovery. Understanding bodies, including those coded as eating disordered, as 'political spaces' (spaces marked by contested power relations; Wright \& Harwood, 2009, p. 12 ), we can interrogate how people come to learn ways to act with and on their bodies, including in eating disorder recovery. Lupton (2013) suggests that biopower plays a role in how we understand and treat both fat and eating disordered bodies. Bodies that fall on either side of the "healthy norm' are represented as in need of 'fixing', often de-feminized and asexualized or hyperfeminized and hypersexualized, and portrayed as freakish, abnormal, emotionally damaged and irrational. People may selfmonitor in an attempt to enact the kinds of bodies that are marked as preferred. In a culture that prizes thinness and toned bodies but simultaneously peddles consumer products encouraging excess, it can be difficult for anyone to decipher how they might go about attaining a body marked as healthy and ideal. As Aphramor and Gingras (2009) suggest, we 
are faced with distinct and often incongruous sets of instructions for how to manage our bodies; eating disordered behaviours might be (at least partially) understood as an attempt to tread the thin line between restraint and indulgence subtly endorsed in our society. In this article, we explore the possibility of a biopedagogy of eating disorder recovery, grounded in prescriptions for health designed to be life sustaining, but subtly holding individuals in recovery to (perhaps unattainable) standards that may feel constraining as they move through their lives in bodies that defy categorization.

\section{METHODOLOGY: STORYING EATING DISORDER RECOVERY}

\section{Data collection and analysis}

We recruited participants using flyers in public spaces and social media, specifying that we were seeking women who had experienced disordered eating, that diagnosis was not a criterion for inclusion and that we welcomed unique stories. We aimed to better understand the embodied, socioculturally situated stories of young women in recovery; accordingly, we used purposeful sampling to obtain 'information-rich cases for study' (Patton, 2002, p. 230) rather than seeking a representative sample. The first author conducted interviews using a conversational style, following a semi-structured interview guide but taking participants' lead. Key questions probed participants to reflect on their experiences of having (e.g. 'What did having an eating disorder mean to you? How did it feel, to you?') and recovering from an eating disorder/disordered eating (e.g. 'What does the word recovery mean to you?'). We performed narrative thematic analysis 
(Riessman, 2007) on interviews with 10 young women 'in recovery'1 1 from eating disorders/ disordered eating. Narrative thematic analysis focuses primarily on the content of the data (what is said); unlike other types thematic analysis, however, the story itself is the unit of analysis, rather than being parsed into smaller nodes for crosscutting themes (Riessman, 2007). Our approach to narrative thematic analysis may also be termed 'critical', because of our integration of critical feminist theories, new materialist and body becoming feminist frameworks (Grosz, 1994; Rice, 2014, in press), into analysis to explore how themes interact with broader systemic and bodily imperatives. We used our theoretical lenses to guide coding and analysis in order to respond to our research questions: We were interested in how dominant biopedagogies come to intersect with prescriptions offered to those in recovery. These feminist theories encourage us to unpack how people experience their bodies in social context.

Rather than theorizing environments as 'acting on' bodies, new materialist and body becoming theorists explore how bodies themselves have agency, intertwining with social contexts in mutually impactful ways. For example, Grosz (1994) describes body-environment interaction as a Mobius strip: bodies are both produced by and produce history and culture. In our analysis, this body-culture interaction became important in order for us to theorize how embodiments might become caught at the intersection of multiple prescriptions for health. These frameworks presented us with the opportunity to recognize that while prescriptions for health have impacts

\footnotetext{
1 'In recovery' has been chosen to articulate the phase at which these young women found themselves in their lives. We have chosen this terminology to maintain consistency throughout the manuscript, but it should be noted that participants did not unanimously self-define as 'in recovery'. Some identified as 'recovered' and others as 'in remission', and others preferred not to use a label.
} 
on bodies, bodies have agency and may conform to or defy prescriptions for health as people come to interact with (resist and take up) these prescriptions and imperatives in novel ways. We integrated this attendance to both context and mind/body agency into our use of narrative thematic analysis by focusing on participants' identification of how standards and discourses, as well as their psychic energies and corporeal propensities, facilitated and/or constrained their embodiment of recovery.

Transcription is the first step toward analysis in Riessman's narrative analysis; it allows researchers to become more familiar with participants' stories, including areas of convergence and divergence between them (Riessman, 2007). Accordingly, the first author tran-scribed all interviews using a detailed orthographic style (indicating pauses and verbal emphasis in addition to transcribing words), checking each a second time for accuracy. The first author also wrote over 60 memos to bracket personal and theoretical observances about the data, taking a critical self-reflexive approach (Rice, 2009) and noting her reactions to and initial observations about the data. We coded data using Dedoose, an online qualitative analysis programme. The first author engaged in open coding, identifying and labelling parts of participants' stories that could feature as narrative themes. We then analysed these codes for those that featured prominently and those that often overlapped, and their location in participants' stories. We created themes from codes based on frequent and co-occurring codes, attending to both similarities and differences across and within participants' accounts. All data collection and analysis were conducted in compliance with an ethics protocol reviewed and approved by the University of Guelph's Research Ethics Board. 


\section{Participants}

Ten young women participated in narrative interviews. Three of these women also participated in a 3-day digital storytelling workshop, where they created short films about their experiences, the results of which will be written up in a separate paper. This workshop acted as a form of member checking: As a part of its development, we created a curriculum based in part on preliminary results of analysis of interview data. Workshop participants (a subset of interview participants) offered thoughts on preliminary data analyses, which informed continued analysis. Participants ranged in age from 20 to 31 (average 25). All were university-educated. Eight had been diagnosed with anorexia nervosa and three with bulimia nervosa (not mutually exclusive; some experienced diagnostic crossover). Two participants had not received formal diagnoses, although both claimed a clinical label, one bulimia nervosa and the other eating disorder not otherwise specified. Five participants identified as Caucasian, two as Jewish, two as European and one as Black Caribbean/middle eastern/White. Seven participants identified as heterosexual; three participants viewed sexuality on a continuum, one identifying as a lesbian in a relationship with a heterosexual man, one as fluid and married to a man and one as mostly heterosexual. We report these complex self-identifications to underscore how even within a group considered homogenous, participants claim diverse spaces of belonging.

\section{RESULTS: MAPPING THE TERRAIN OF EATING DISORDER RECOVERY}

Participants' stories reflected deeply situated understandings of the 
experience of having and recovering from eating disorders. Despite individual differences, emerging themes painted an overall picture of the complex, multi-faceted terrain of eating disorders and recovery. The following themes should be interpreted with an acknowledgment of the various degrees to which participants both adopted and complicated, in Foucauldian terms, 'dominant' and 'subjugated' discourses around eating disorders and recovery.

\section{Doctor's orders: Standards for recovery}

Participants reported that the state or label of recovered constitutes a set of expectations and standards. Instructions for living recovered should not be read as wholly problematic; in some ways, having a set of criteria (e.g. a meal plan to follow and a weight standard to attain) kept participants 'in check' or healthy. However, the recovered label can also uphold new instructions for living that contradict health promotion messages for the general public (e.g. following a highly caloric meal plan in an environment that encourages caloric restriction). In the subsequent quotes, names have been changed to pseudonyms.

\section{Materiality of eating disorders and recovery}

Participants were fluent in biomedical discourses, using them to help make sense of their experiences of having eating disorders. We refer to this theme as 'materiality', bringing in the ways in which participants both took up and challenged recovery biopedagogies. Biomedical explanations are rational; they reinforce the mind-body split so often taken for granted in health discourses. We are accustomed to understanding bodies as fixed and bounded, with mind in control of body. When this control is absent, the individual is assumed to be suffering from a deficit of mind in need of cure 
(Shildrick, 1997). Participants' stories echoed with this dualism: For example, they often specifically referred to 'rewiring' their brains. Naming the brain as the element germane to the technology of cure offers insight into how it may be (at times) conceptualized as an entity not 'of the body'.

Understanding eating disorders and the process of recovery through a biomedical lens helped Andie to accept slips or relapses along the way:

I had to work a lot with my individual therapist about taking those moments as learning experiences and taking pride in the little things that I could do to delay binge eating, viewing those as ways that I was breaking the habitual nature to continually engaging in the eating disorder and reminding myself of, I study addiction. Of course I'm going to relapse [...] The cues associated with binge food intake, they're going to trigger the compulsion to engage in it again. It's extreme. What makes my brain so different from the norm? (Andie)

For Andie, positioning her brain as normal and these behaviours as scientifically logical helped her to forgive herself for 'resorting to' eating disordered symptoms. Other participants used biomedical framing to highlight how their symptoms were logical coping mechanisms. Here, eating disorder behaviours are scientifically explained responses to stressful stimuli (see also Easter, 2012). This attribution nuances biomedical discourses surrounding eating disorders: Although they locate the problem within the individual (a common post- structuralist critique of such discourses), they may also downplay moralizing construction of behaviours around food and exercise.

Participants also subtly endorsed biomedical discourses around 
disordered eating shared with them by medical 'experts'. They recounted stories through words and instructions that closely mirrored medical professionals' instructions for eating disorder recovery:

I think I had been malnourished for a long time... one of my friends who is in med school- she's a psychiatrist [...] also had an eating disorder at one point- [she] sent me some research articles which were saying that being malnourished can actually start or perpetuate [eating disorders] so I'm like, well that kind of makes sense! They always said to us no matter what after treatment we just had to keep eating and I always questioned why, and they said it affects your brain. (Gabrielle)

Again, one of the most compelling things about biomedical discourses was their overt rationality. They helped participants make sense of behaviours socially coded as irrational and extreme; these reasonable, logical attributions acted as currency in stories about recovery. Participants' strategic adoption of biomedical explanations may be significant as it unearths how such discourses usefully explicate something of the materiality of bodily cravings and drives, albeit in potentially reductive ways, but also in ways that resonate with individuals' experiences and that have eluded post-structural and other constructionist accounts.

\section{Beyond biometrics}

Participants also critiqued biometrics_-body characteristics and biomedical criteria related to eating and weight-as the main markers of recovery. For example, many participants had reached a healthy body weight in treatment programmes, meeting the minimum standard criteria for 
discharge. However, when they perceived weight gain to be the primary (or only) focus of a treatment programme, participants questioned the longterm efficacy of treatment. Participants suggested that BMI or weight gain might be used as proxies for recovery but say little about the psychological well-being (or even physical well-being) of individuals in recovery (see also Boughtwood \& Halse, 2008). These observations echo literature that explores the limits of treatment focused primarily on weight restoration (e.g. Boughtwood \& Halse, 2008, 2010; Gremillion, 2003). Although participants did not approach weight gain only as a 'means to an end' (i.e. to get out of the hospital and recommence self-starving or bingeing/purging; Boughtwood \& Halse, 2010), neither did they perceive themselves to have reached a point of 'full recovery' upon reaching discharge weight.

Boughtwood and Halse (2008) suggest that 'normality' may be exceedingly difficult to achieve in such a highly surveilled context; participants' accounts agreed with this assessment.

All participants problematized the idea that recovery could be reached by refeeding alone, even suggesting that such an approach set them back in their recovery journey.

When I was seeking treatment here in Ontario, a huge part of it was placed on the refeeding process, and getting me to a healthy weight. Which is understandable, but it almost felt like they were rushing into that part of that and didn't have a game plan for what help I was going to be receiving after I got to a healthy weight $[\ldots]$ I knew that it was going to take me a lot longer to mentally be healthy again. It's one thing to gain weight and be sent on your way, and then come home 
and start planning that you're going to start losing weight again, which is exactly what I did after treatment. So, I think you do have to worry about the physical aspects, obviously, but you have to come at the emotional aspect just as hard. (Alice)

Meal plans also occupied an ambiguous place in participants' accounts. While they provided the women with the ability to take calculated risks, they often failed to take into account cultural practices and familial norms around food. Gabrielle, a young Jewish woman, noted feeling upset when she was not allowed to dress her holiday meal in accordance with Jewish traditions.

When they served baked potatoes and pork and beans and I put my applesauce on my baked potato they called it a weird food combination, and we weren't allowed to eat weird food combinations. And I was like how is that weird, we put applesauce on latkes, and latkes are made of potatoes, and everyone said what's a latke? (Gabrielle)

The notion of normal eating loomed large in participants' accounts as they attempted to disentangle eating plans prescribed in treatment in a Western sociocultural milieu from their own family cultural practices surrounding eating.

\section{Embodying counter-culture}

Discourses around recovery, whether prescribed by clinicians or selfimposed, intersect with dominant biopedagogies for bodily management in ways that participants described as constraining, conflicting and overwhelming. The limits of biomedical discourses on recovery emerge 
when juxtaposed with prescriptions for nor- mal eating and healthy living sent to the broader population. Participants felt caught between opposing discourses for body management: those spoken by family, friends, media and others and those prescribed in healthcare settings designed to facilitate recovery. Participants expressed feeling different from people who had never had eating disorders in how they were instructed to be around food. This was especially challenging once their bodies had crossed over into a place to being read as normal: No longer marked by the physicality of 'having an eating disorder', they were assumed to be in thrall to the same biopedagogical instructions as those who had never had eating disorders, a bind that weighed heavily on many.

Some participants described following a 'counter-cultural' recovery regimen as extremely difficult, even 'mind blowing'.

When I was in the first stage of recovery, I couldn't believe the things they asked my parents to do like put melted butter in hot chocolate to boost the calories, you know? Dessert every day right after dinner and then a snack after that. Mind blowing. It's very opposite [to] 'make healthy choices, follow Canada's food guide', that kind of thing and l'd be like well, sorry, l've got to go home and eat my chocolate cake and Ensure! It made it very hard to believe the doctors. I was convinced they were wrong because they were saying the opposite of what everything else was saying. And that made it really hard to follow through. (Ella)

Enacting counter-culture entailed acknowledging the prescriptions given to the general population (e.g. Canada's Food Guide) and 
recognizing that 'recovery as prescribed' often involved doing the opposite (e.g. eating chocolate cake and drinking Ensure). On the other hand, meal plans prescribed by dieticians could also act as evidence and ammunition for combatting dominant discourses surrounding weight and food, giving participants evidence for their enactment of counter-culture.

Participants often felt watched (surveilled) both in and beyond treatment settings. Describing her experiences at a non-specialist treatment centre, Katie reflects on this surveillance:

I felt like I was treated like a- not even a child- like a case study, it was very odd. I remember this one woman, giving me my night snack or whatever [...] she just sat there and looked at me. Sat there and looked at me with this sandwich, and I was terrified of eating this stupid sandwich, and she was so mesmerized by this anorexic girl eating this sandwich and I was mortified. It was awful. (Katie)

Katie's experiences of discomfort underscore the degree to which disordered eating is socially coded as extreme, an object of fascination and confusion (Lupton, 2013; Warin, 2010). This surveillance does not end in the treatment setting; participants' histories of eating disorders coloured others' interpretations of their behaviours long after treatment. Participants problematized the expectation that they should be ok with eating foods that others would be praised for not eating. Their stories illustrate how behaviours and actions that would otherwise be read as health conscious and positive may be read as elements of continued pathology in individuals who have recovered from eating disorders.

It's like you have to be ok with eating everything [...] for other people 
it's some level of 'oh well that's kind of oily I don't want to eat that', but for you it's 'that's your eating disorder talking'. (Margot)

In Margot's quote, she also notes how others draw upon an externalizing discourse ('that's your eating disorder talking'). Externalization, or the practice of separating the eating disorder from the person, is a discourse often used in narrative therapy to avoid conflating people with their eating problems and to enable a re-storying of experiences (Maisel, Epston, \& Borden, 2004). Externalization is also increasingly being taken up in mainstream treatment modalities, wherein clinicians urge a separation between 'Ed voice' and patient's voices. Here, Margot responds to such externalization in a critical way, taking issue with how those who knew that she had previously experienced an eating disorder continued to conflate restrained eating with the disorder, a move that marked her as potentially permanently disordered and as unable to engage in prescriptions for healthy eating given to the broader public. Taken to its extreme, externalization rendered Margot disordered from the vantage point of treatment discourses that saw any restriction as symptomatic of eating distress. Yet in failing to avoid too much fat, she was simultaneously marked as unhealthy by healthy eating discourses that advocate normative restraint.

Meal plans and other metrics for health are evidently not simply 'good' or 'bad' for eating disorder recovery; different social actors, including participants, doctors, dieticians, friends and family members, use these discourses in alternatively problematic and helpful ways. Participants' accounts of difficulties with biopedagogies surrounding weight and food 
gesture toward some of the ways in which recovery regimes encouraged forms of bodily self-surveillance that both mimicked and inverted the eating disordered mindset/behaviour from which participants were attempting to recover. Their stories unveil how something they knew to have helped to save their lives became problematic as recovery progressed. Prescriptions and instructions for recovery served fluid and dynamic roles in participants' lives and were more or less helpful depending on where a participant was in her recovery and life. Rather than completely abandoning these discourses, participants discovered ways of working both within and beyond dominant discourses and prescriptions for recovery.

Embodying recovery

Participants hesitated to put a timeline on recovery or to claim that there was a moment when they knew themselves to be recovered. Thinking about 'full recovery' could be overwhelming, rather than a helpful goal:

Thinking so much about full recovery is kind of like only being able to run one kilometer and thinking about when you can run a whole marathon. It's like that's too big and it seems so- it can seem motivational to some people like 'yes I want to get to that point where I can run a full marathon,' but to other people it seems ridiculously intimidating. (Margot)

Importantly, reticence to claim full recovery did not mean that participants were 'giving up' or settling for 'less than'. Counter to Shohet's (2007) suggestion that claiming 'full recovery' is necessary for maintaining a stable life, interviewees noted that their lives no longer revolved around food and weight, even if they refused to claim the recovery label. Some 
participants described the label as imbued with expectations of invulnerability, choosing instead to conceptualize recovery as a dynamic process (see also Federici \& Kaplan, 2008). Some described the idea of returning to a state prior to the eating disorder as unrealistic and avoided describing recovery as one self as replacing another. Instead, participants articulated poly-vocal selves, in the sense that one self does not come to replace another, but that people draw upon different parts of their subjectivities in different contexts (Saukko, 2008) in the process of becoming (Rice, 2014, in press). For example, Maya described 'living her recovery every day':

The term recovery to me is tricky because I consistently think of recovery as a process, not something that I necessarily fully achieve but something that I'm continuing to maintain. It's kind of like an action: I'm living my recovery every day, rather than I've reached this one moment saying ok l'm better. Because I feel [that] denies you the space to say that it's hard. To say that it's actually difficult sometimes. To say that sometimes I eat too much pizza and I feel that thing in my chest and I could go and throw up but I don't. Perhaps it would be in those moments I feel that I'm recovered, when I don't do it. That I choose not to, that I make the choice not to. Because it's ok. So in a psychiatric medical model I might not necessarily fully be recovered, because I still think about it, but I don't I don't do it anymore. (Maya) Recovery, here, is the decision to act opposite in the moments where she knew she would previously have engaged in symptoms. For Maya and others, neither the label of eating disordered nor recovered fully 
encapsulated their embodied experiences.

Authenticity and invisibility/visibility

Participants reflected on their experiences in relational ways. Their stories were rich with descriptions of interactions with doctors, friends and family members who interpreted their illness or lack thereof by observing their bodies. These interactions informed participants' interpretations of the legitimacy of their experiences. Often, participants' physical appearance was at odds with their sense of psychological well-being. For example, maintaining a healthy body weight could actually be a side effect of engaging in bingeing and purging. At times, they described actively avoiding letting their bodies read as disordered by not 'fully' acting out disordered behaviours.

I never really had the guts to fully go through with a big enough cry for help because I knew that it would embarrass my mother and I just I really didn't want her to be embarrassed on top of every-thing else. (Maya)

Not having others notice or acknowledge eating disorder behaviours often led to participants continuing to discount feelings of distress; seeing their bodies through the eyes of others fed into feelings of not being sick enough to seek treatment. Disturbingly, physicians sometimes replicated these opinions and readings of their bodies:

I went to the doctor to get bloodwork done and the doctor's like... you're fine. But you have the BMI of an anorexic. That started getting the wheels turning that maybe something is wrong. (Andie) In the eyes of others in participants' lives, recovery was strongly 
linked to achieving a healthy weight, sometimes to the extent that it eclipsed the other elements of recovery participants felt were important. When body weight alone becomes the lens through which people determine whether or not someone has a 'legitimate' eating disorder, those whose bodies never conform to the stereotype of an 'eating disordered body' are left in an interstice between well and unwell. Individuals who do not 'need' to gain weight in recovery may feel that their struggles and ensuing recovery are not authentic or legitimate in the face of strong messages from others who never read their bodies as disordered in the first place.

Even when their disorders were coded as legitimate problems via diagnosis, some participants struggled with perceiving certain elements of their treatment protocols as helpful. Still, we cannot ignore the fact that the participants in this study identified as being well. Therefore, something must have been helpful, along the way. Participants were aware of sometimes needing to critique the very things that had helped them to get where they were.

\section{Limitations}

This study gestures toward a biopedagogy of recovery that may help us to understand the diverse, embodied experiences of young women in eating disorder recovery. However, we recognize its limitations. First, our sample size was small and relatively homogenous. Participants complicated the boxes in which we place research participants which is, itself, an interesting finding. Still, drawing conclusions about the pervasiveness of themes amongst diverse individuals with eating disorders would be difficult. Future research might seek to replicate these findings in a 
diverse group (e.g. non-heterosexual individuals, men, trans people, individuals of various ethnicities, and socioeconomic statuses). A more representative sample might nuance the concept of a biopedagogy of recovery and reveal diverse impacts on marginalized individuals. Further, participants self-selected into this study. Participants self-identified as in recovery but also problematized this category as the interviews progressed, often struggling to put timelines on their experiences of disordered eating and recovery. While this complicated the linearity of the participants' stories, we interpreted their narratives as they were told, recognizing that all stories are shaped for audiences, including researchers, and privileging depth of embodied experience over timeline accuracy (Riessman, 2007).

\section{Conclusions}

As Bardone-Cone et al. (2010) note, there is significant divergence in conceptualization of recovery across the clinical literature. Aligning ourselves with efforts to attend to participants' perspectives on recovery (e.g. Darcy et al., 2010; Dawson et al., 2014; Federici \& Kaplan, 2008; Hay \& Cho, 2013; Ison \& Kent, 2010; Malson et al., 2011; Shohet, 2007), we examined how participants' accounts replicate and/or challenge clinical and popular recovery discourses embedded in sociocultural context. The detrimental effects of biopedagogies of weight and shape are well acknowledged in the critical psychology literature (Evans \& Rich, 2011; Rice, 2009, 2014). In a society in which bodies are imbued with moral characteristics, including ill/health, fitness/unfitness or productivity/laziness, prescriptions for health can, paradoxically, have deleterious effects. Those whose bodies are coded as overweight or obese may avoid the very health 
promoting behaviours they are 'supposed to' engage in or may experience distress-inducing stigma (Rice, 2007). Through biopedagogies, social actors urge consumption and also restraint, excess and also control.

We propose that a similar but opposite biopedagogy may be operating for individuals in eating disorder recovery, grounded in potentially health-enhancing, even life-saving techniques and technologies of cure. While prescriptions for recovery may keep participants 'in check', they may also reinforce problematic relationships with food and control. Where following a meal plan could be viewed as compliance with recovery discourses while in treatment, at some point, participants were expected to shuck off the rigidity surrounding food imposed both during the eating disorder and in treatment. As in Gremillion's (2003) analysis of gender and power in treatment settings, compliance is valued to a certain extent, until patients are expected to exercise individualism and 'rebel'-within set limits. Participants expressed significant frustration with being held to different standards than others in their lives. The need to be invulnerable, and 'ok with eating anything' led to dis- comfort with the very label of recovery and its association with unrealistic standards. Deepening our collective understanding around a biopedagogy of eating disorder recovery may help us to better support individuals as they move forward in their lives in ways that may be counter to dominant culture.

Within the narrative therapy tradition, externalizing the problematic aspects of eating disorder subjectivities represents a critical therapeutic response to eating disorders. However, participants' experiences suggest that clinicians should be cautious about taking a wholly oppositional or 
combative stance toward the eating disordered self. This position does not create room for people to make mistakes in recovery without judgement or shame, to recuperate aspects the mindset coded as eating disordered (with its emphasis on commitment, productivity and perfectionism) that may be valuable to them and to see themselves as 'normal' if we accept that normal within the current sociocultural climate means some degree of weight consciousness and eating restraint. Further, if we begin with the premise that embodied subjectivities are produced in and through cultural discourses that circulate at all levels in society (including treatment contexts), then approaching recovery as a process whereby persons change their relationship to the mindset coded as eating disordered rather than severing that relationship entirely may allow for a less moralizing construction of behaviours around food and exercise, for people to claim aspects of their selves culturally and clinically associated with eating disorders (such as retaining some of their drive for perfectionism or productivity) and, hence, to begin to imagine themselves as recovered.

Normal eating is difficult to define in a society that routinely promises individuals that they can lose weight by subscribing to the newest dietary craze; this difficulty is exacerbated when applying the construct to individuals recovering from eating disorders. Becoming attuned to bodily surveillance (from without and within) helps to open the possibility of supporting eating disorder recovery. Others have begun to unearth the complexity of recovery from eating disorders, suggesting that recovery entails achieving holistic wellness (Jenkins \& Ogden, 2012) or reflection (Dawson et al., 2014). Here, we draw attention to a sociocultural context that may make getting to this place difficult or may limit the degree to which 
people may be able to imagine recovery (Malson et al., 2011). We have highlighted the counter-cultural nature of recovery, the subtle standards of invulnerability and transcendence held within the concept of recovery, and the paradoxical control and surveillance within and beyond treatment contexts. Being conscious of the degree to which participants might feel constrained by bodily moral imperatives and norms may help to guide the creation of appropriate supports for people in recovery.

\section{ACKNOWLEDGEMENTS}

This research was supported by the Ontario Women's Scholar Award through the Ontario Ministry of Health and Longterm Care. The views expressed in the material are the views of the authors and do not necessarily reflect those of the Ministry of Health and Long-Term Care. The authors wish to thank participants for sharing their stories.

\section{REFERENCES}

Aphramor, L., \& Gingras, J. (2009). Sustaining imbalance: Evidence of neglect in the pursuit of nutritional health. In S. Riley, M. Burns, H. Frith, S. Wiggins \& P. Markula (Eds.), Critical bodies: Representations, identities and practices of weight and body management (pp. 155174). Hampshire, UK: Palgrave Macmillan.

Bardone-Cone, A., Harney, M. B., Maldonado, C. R., Lawson, M. A., Robinson, P., Smith, R., \& Tosh, A. (2010). Defining recovery from an eating disorder: Conceptualization, validation, and examination of psychosocial functioning and psychiatric comorbidity. Behavioural Research and Therapy, 48, 194-202. 
Boughtwood, D., \& Halse, C. (2008). Ambivalent appetites: Dissonances in social and medical constructions of anorexia nervosa. Journal of Community and Applied Social Psychology, 18, 269-281.

Boughtwood, D., \& Halse, C. (2010). Other than obedient: Girls' constructions of doctors and treatment regimes for anorexia nervosa. Journal of Community and Applied Social Psychology, 20, 83-94.

Darcy, A. M., Katz, S., Fitzpatrick, K. K., Forsberg, S., Utzinger, L., \& Lock, J. (2010). All better? How former anorexia nervosa patients define recovery and engaged in treatment. European Eating Disorder Review, $18,260-270$.

Dawson, L., Rhodes, P., \& Touyz, S. (2014). "Doing the impossible": The process of recovery from chronic anorexia nervosa. Qualitative Health Research, 24, 494-505.

Easter, M. M. (2012). Not all my fault: Genetics, stigma, and personal responsibility for women with eating disorders. Social Science \& Medicine, 75, 1408-1416.

Evans, J., \& Rich, E. (2011). Body policies and body pedagogies: Every child matters in totally pedagogised schools? Journal of Education Policy, 26, 361-379.

Federici, A., \& Kaplan, A. S. (2008). The patient's account of relapse and recovery in anorexia nervosa: A qualitative study. European Eating Disorders Review, 16, 1-10.

Foucault, M. (1979). Discipline and punish: The birth of the prison. New York: Vintage Books. 
Fullagar, S. (2009). Governing healthy family lifestyles through discourses of risk and responsibility. In J. Wright \& V. Harwood (Eds.), Biopolitics and the 'Obesity Epidemic': Governing bodies (pp. 108-126). New York: Routledge.

Gremillion, H. (2003). Feeding anorexia: Gender and power at a treatment centre. Durham: Duke University Press.

Grosz, E. (1994). Volatile bodies: Toward a corporeal feminism. Bloomington, IN: Indiana University Press.

Halse, C. (2009). Bio-citizenship: Virtue discourses and the birth of the biocitizen. In J. Wright \& V. Harwood (Eds.), Biopolitics and the 'obesity epidemic': Governing bodies (pp. 45-59). New York: Routledge.

Harwood, V. (2010). Mobile asylums: Psychopathologisation as a personal, portable psychiatric prison. Discourse: Studies in the Cultural Politics of Education, 31, 437-451.

Hay, P. J., \& Cho, K. (2013). A qualitative exploration of influences on the process of recovery from personal written accounts of people with anorexia nervosa. Women \& Health, 53, 730-740.

Ison, J., \& Kent, S. (2010). Social identity in eating disorders. European Eating Disorders Review, 18, 475-485.

Jenkins, J., \& Ogden, J. (2012). Becoming "whole" again: A qualitative study of women's views of recovering from anorexia nervosa. European Eating Disorders Review, 20, e23-e31.

LaMarre, A., \& Rice, C. (In press). Unrecoverable? Prescriptions and possibilities for eating disorder recovery. In N. Khanlou \& B. Pilkington 
(Eds.), Women's mental health: International perspec- tives on resistance and resilience in community and society.

Lupton, D. (2013). Fat. New York: Routledge.

Maisel, R., Epston, D., \& Borden, A. (2004). Biting the hand that starves

you: Inspiring resistance to anorexia/bulimia. New York: W.W. Norton.

Malson, H., Bailey, L., Clarke, S., Treasure, J., Anderson, G., \& Kohn, M.

(2011). Un/imaginable future selves: A discourse analysis of inpatients' talk about recovery from an "eating disorder". European Eating Disorders Review, 19, 25-36.

Noordenbos, G., \& Seubring, A. (2006). Criteria for recovery from eating disorders according to pa- tients and therapists. Eating Disorders, 14, 41-54.

Patton, M. (2002). Qualitative research and evaluation methods (3rd edn). Thousand Oaks, CA: Sage.

Rail, G. (2012). The birth of the obesity clinic: Confessions of the flesh, biopedagogies and physical culture. Sociology of Sport Journal, 29, 227-253.

Rance, N. M., Clarke, V., \& Moller, N. P. (2014). "If I see somebody... I'll immediately scope them out": Anorexia nervosa clients' perceptions of their therapists' body. Eating Disorders, 22, 111-120.

Rice, C. (2007). Becoming the fat girl: Emergence of an unfit identity. Women's Studies International Forum, 30, 158-174.

Rice, C. (2009). Imagining the other? Ethical challenges of researching and writing women's embodied lives. Feminism \& Psychology, 19, 245- 
266.

Rice, C. (2014). Becoming women: The embodied self in image culture.

Toronto: University of Toronto Press.

Rice, C. (In press). Re-thinking fat: From bio- to body becoming pedagogies. Cultural Studies $<=>$ Critical Methodologies.

Riessman, C. K. (2007). Narrative methods for the human sciences. Newbury Park, CA: Sage.

Saukko, P. (2008). The anorexic self: A personal, political analysis of a diagnostic discourse. New York: SUNY Press.

Shildrick, M. (1997). Fabrica(tions): On the construction of the human body. In M. Shildrick (Ed.), Leaky bodies and boundaries: Feminism, postmodernism and (bio)ethics (pp. 13-61). London: Routledge.

Shohet, M. (2007). Narrating anorexia: "full" and "struggling" genres of recovery. Ethos, 35, 344-382.

Warin, M. (2010). Abject relations: Everyday worlds of anorexia. Piscataway, NJ: Rutgers University Press.

Wright, J., \& Harwood, V. (Eds.) (2009). Biopolitics and the "obesity epidemic": Governing bodies. New York, NY: Routledge. 